Document downloaded from:

http://hdl.handle.net/10251/64692

This paper must be cited as:

Budzko, D.; Cordero Barbero, A.; Torregrosa Sánchez, JR. (2015). A new family of iterative methods widening areas of convergence. Applied Mathematics and Computation. 252:405417. doi:10.1016/j.amc.2014.12.028.

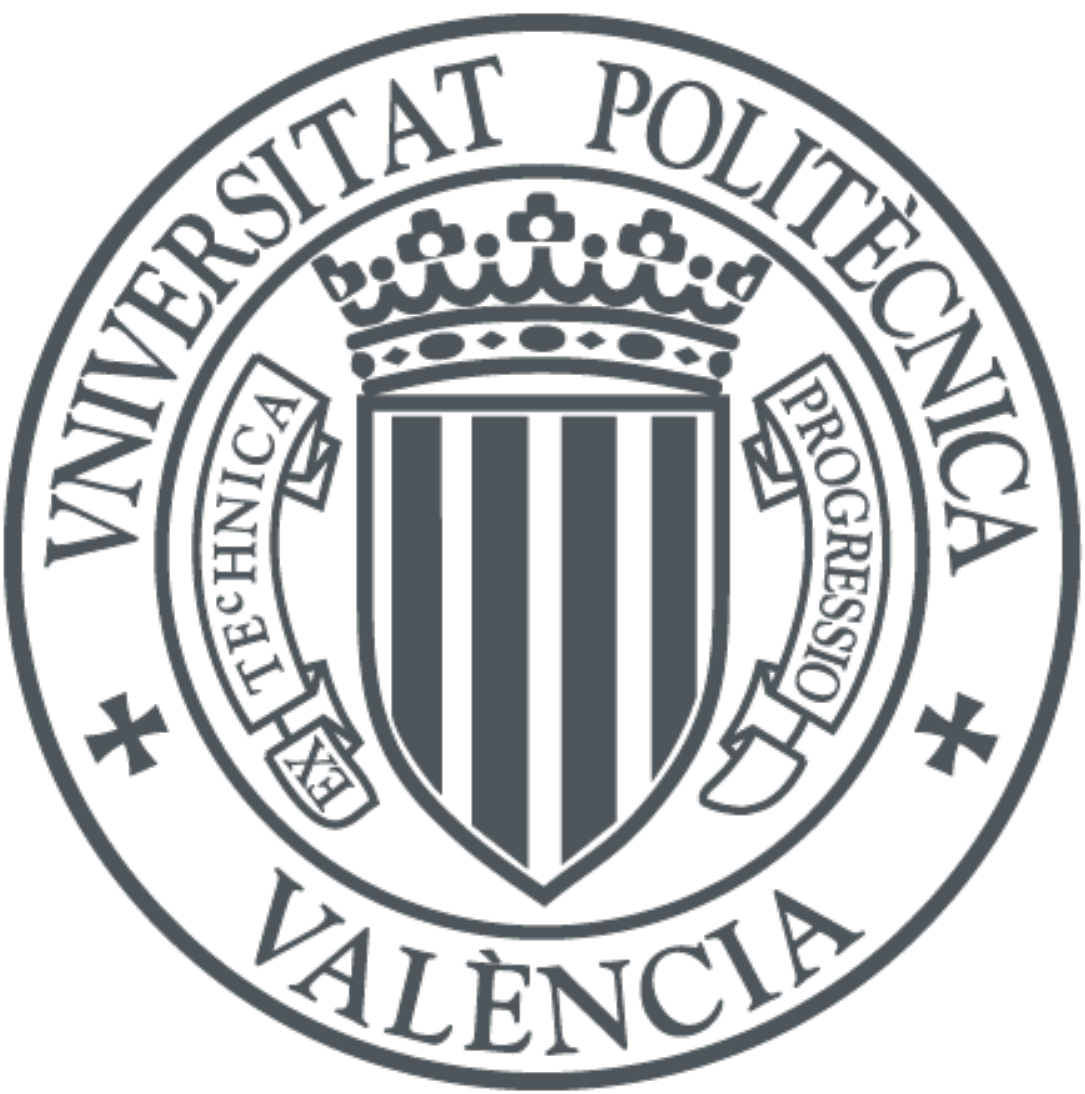

The final publication is available at

https://dx.doi.org/10.1016/j.amc.2014.12.028

Copyright Elsevier

Additional Information 


\title{
A new family of iterative methods widening areas of convergence
}

\author{
Dzmitry Budzko $^{\mathrm{a}}$, Alicia Cordero ${ }^{\mathrm{b}, *}$, Juan R. Torregrosa ${ }^{\mathrm{b}}$ \\ ${ }^{a}$ Department of Informatics and Computer systems \\ Brest State University \\ Kosmonavtov Boulevard 21, 224016 Brest, Belarus \\ ${ }^{b}$ Instituto de Matemática Multidisciplinar \\ Universitat Politècnica de València \\ Camino de Vera s/n, 46022 València, Spain
}

\begin{abstract}
A new parametric class of third-order iterative methods for solving nonlinear equations and systems is presented. These schemes are showed to be more stable than Newton', Traub' or Ostrowski's procedures (in some specific cases), and it has been proved that the set of starting points that converge to the roots of different nonlinear functions is wider than the one of those respective methods. Moreover, the numerical efficiency has been checked through different numerical tests.
\end{abstract}

Keywords: Nonlinear systems, iterative methods, basin of attraction, dynamical plane, convergence domain, order of convergence, divided difference

\section{Introduction}

Nonlinear equations or systems are usually involved in different problems in science and engineering. The analytical solution of these kind of problems is difficult and often iterative methods are used in order to estimate the solutions.

In the last decade, many multipoint Newton-type iterative schemes for solving nonlinear equations have appeared in the literature. A good survey about them can be found in [1]. Usually, these variants of Newton's method are designed to improve the original scheme in terms of order of convergence. However, they have some drawbacks: many of them are not applicable to nonlinear systems and high-order methods have quite reduced areas of convergence. We give emphasis, not as much to the order of convergence but to the region of good starting points of the methods and in the extension to nonlinear systems. So, our aim is to design a parametric family of iterative methods with wider areas of convergence than other known schemes and its generalization to multivariate cases.

In this work, we present a family of uniparametric two-point iterative procedure for solving the nonlinear equation $f(x)=0$. It uses a damped Newton in the first step (acting as a predictor) and the corrector step is defined as a Newton-type scheme in which three functional evaluations are used. This corrector step is inspired in some modifications of Newton's method proposed by V.V. Ermakov and N.N. Kalitkin in [2]. Specifically, the authors presented a damped Newton scheme

$$
x_{k+1}=x_{k}-\beta_{k} \frac{f\left(x_{k}\right)}{f^{\prime}\left(x_{k}\right)}, \quad k=0,1, \ldots
$$

\footnotetext{
The project has been funded with support from European Commission. This research was supported by Ministerio de Ciencia y Tecnología MTM2011-28636-C02-02

${ }^{*}$ Corresponding author

Email addresses: dzmitry.budzko@gmail.com (Dzmitry Budzko), acordero@mat.upv. es (Alicia Cordero ), jrtorre@mat . upv.es (Juan R. Torregrosa )
} 
where $\beta_{k}=\frac{\left\|f\left(x_{k}\right)\right\|^{2}}{\left\|f\left(x_{k}\right)\right\|^{2}+\left\|f\left(x_{k}-\frac{f\left(x_{k}\right)}{f^{\prime}\left(x_{k}\right)}\right)\right\|^{2}}$.

We propose the following iterative expression:

$$
\begin{aligned}
y_{k} & =x_{k}-\alpha \frac{f\left(x_{k}\right)}{f^{\prime}\left(x_{k}\right)}, \quad k=0,1, \ldots \\
x_{k+1} & =x_{k}-\frac{f\left(x_{k}\right)^{2}}{b f\left(x_{k}\right)^{2}+c f\left(y_{k}\right)^{2}} \frac{f\left(x_{k}\right)}{f^{\prime}\left(x_{k}\right)},
\end{aligned}
$$

where $\alpha, b$ and $c$ are parameters. In the rest of the paper, we will denote this method by PM.

We prove that, under some conditions, the local order of convergence of the elements of the family is three. We can also extend this family for solving nonlinear systems $F(x)=0$, holding the order of convergence.

We will compare the proposed schemes, in terms of the wideness of the regions of converging initial points, with the well-known Newton's method, whose iterative expression is

$$
x_{k+1}=x_{k}-\frac{f\left(x_{k}\right)}{f^{\prime}\left(x_{k}\right)}, \quad k=0,1, \ldots
$$

It is known that this scheme converges quadratically in some neighborhood of the solution, under standard conditions. In comparisons, we will also use Traub's method (see [3]), which has order of convergence three (as our proposed schemes), whose iterative expression is

$$
\begin{aligned}
& y_{k}=x_{k}-\frac{f\left(x_{k}\right)}{f^{\prime}\left(x_{k}\right)}, \\
& x_{k+1}=x_{k}-\frac{f\left(x_{k}\right)+f\left(y_{k}\right)}{f^{\prime}\left(x_{k}\right)}, \quad k=0,1, \ldots
\end{aligned}
$$

In spite of the lower order of convergence of our proposed schemes, we will also compare them with Ostrowski's procedure [4], which iterative expression is

$$
\begin{aligned}
& y_{k}=x_{k}-\frac{f\left(x_{k}\right)}{f^{\prime}\left(x_{k}\right)}, \\
& x_{k+1}=y_{k}-\frac{f\left(x_{k}\right)}{f\left(x_{k}\right)-2 f\left(y_{k}\right)} \frac{f\left(y_{k}\right)}{f^{\prime}\left(x_{k}\right)}, \quad k=0,1, \ldots
\end{aligned}
$$

and whose order of convergence is four.

Let us note that expressions (1), (3) and (4) can be directly extended to nonlinear systems. On the other hand, recently, iterative expression (5) has been adapted for solving nonlinear systems.

In Section 2 we will analyze the local order of convergence of the designed schemes to find a simple root $\xi$ of a nonlinear equation $f(x)=0$, where $f: D \subseteq \mathbb{R} \rightarrow \mathbb{R}$ is a scalar function on an open interval $D$. Moreover, the procedures will be extended in Section 2.2 for solving systems of nonlinear equations $F(x)=0$, where $F: D \subseteq \mathbb{R}^{n} \rightarrow \mathbb{R}^{n}, n>1$. The dynamical behavior on different interesting equations will be studied in Section 3 , by using distinct values of the parameter. This will allow us to select the most appropriate elements of the class in order to make the numerical test in Section 4, not only with nonlinear equations, but also with some nonlinear systems. Finally, we will state some conclusions and the references used.

\section{Analysis of convergence}

Firstly, we analyze the local order of convergence of the class of methods (2). Afterwards, we extend the mentioned family to nonlinear systems and we also study its convergence. 


\subsection{Study of the scalar case}

In the next result, we show that there are values of parameters $b$ and $c$, depending on $\alpha$, such that the order of convergence of the resulting methods is three.

Theorem 1. Let $\xi \in I$ be a simple zero of a sufficiently differentiable function $f: I \subset \mathbb{R} \rightarrow \mathbb{R}$ in an open interval $I$ and $x_{0}$ an initial guess close enough to $\xi$. The method defined by (2) has third-order convergence if $b=\frac{1-\alpha+2 \alpha^{2}}{2 \alpha^{2}}$ and $c=\frac{1}{2 \alpha^{2}(\alpha-1)}$, where $\alpha \notin\{0,1\}$. The error equation of the method is

$$
e_{k+1}=\frac{\left(-2+2 \alpha+\alpha^{2}\right) c_{2}^{2}+2(-1+\alpha)^{2} c_{3}}{2(-1+\alpha)} e_{k}^{3}+O\left(e_{k}^{4}\right)
$$

where $c_{k}=(1 / k !) \frac{f^{(k)}(\xi)}{f^{\prime}(\xi)}, k=1,2, \ldots$ and $e_{k}=x_{k}-\xi$.

Proof: The proof is based on the Taylor's expansion of the elements appearing in the iterative expression (2).

By using Taylor's expansion around $\xi$, we have

$$
f\left(x_{k}\right)=f^{\prime}(\xi)\left[e_{k}+c_{2} e_{k}^{2}+c_{3} e_{k}^{3}\right]+O\left(e_{k}^{4}\right),
$$

and

$$
f^{\prime}\left(x_{k}\right)=f^{\prime}(\xi)\left[1+2 c_{2} e_{k}+3 c_{3} e_{k}^{2}+4 c_{4} e_{k}^{3}\right]+O\left(e_{k}^{4}\right)
$$

By substituting these expressions in the first step of (2), we obtain

$$
y_{k}-\xi=(1-\alpha) e_{k}+\alpha c_{2} e_{k}^{2}+2 \alpha\left(-c_{2}^{2}+c_{3}\right) e_{k}^{3}+O\left(e_{k}^{4}\right) .
$$

Expanding in Taylor's series $f\left(y_{k}\right)$ around $\xi$,

$$
f\left(y_{k}\right)=f^{\prime}(\xi)\left[(1-\alpha) e_{k}+\left(1-\alpha+\alpha^{2}\right) c_{2} e_{k}^{2}-\left(2 \alpha^{2} c_{2}^{2}+\left(-1+\alpha-3 \alpha^{2}+\alpha^{3}\right) c_{3}\right) e_{k}^{3}\right]+O\left(e_{k}^{4}\right)
$$

and then, combining these expressions,

$$
\begin{aligned}
u= & \frac{f\left(x_{k}\right)^{2}}{b f\left(x_{k}\right)^{2}+c f\left(y_{k}\right)^{2}} \\
= & \frac{1}{b+(-1+\alpha)^{2} c}+\frac{2(-1+\alpha) \alpha^{2} c c_{2}}{\left(b+(-1+\alpha)^{2} c\right)^{2}} e_{k} \\
& +\frac{\alpha^{2} c\left(\left(-\left(-6+6 \alpha+\alpha^{2}\right) b+3(-1+\alpha)^{2}\left(2-2 \alpha+\alpha^{2}\right) c\right) c_{2}^{2}\right)}{\left(b+(-1+\alpha)^{2} c\right)^{3}} \\
& -\frac{\alpha^{2} c\left(2\left(3-4 \alpha+\alpha^{2}\right)\left(b+(-1+\alpha)^{2} c\right) c_{3}\right)}{\left(b+(-1+\alpha)^{2} c\right)^{3}} e_{k}^{2}+O\left(e_{k}^{3}\right) .
\end{aligned}
$$

Therefore, the error at the last step is

$$
\begin{aligned}
x_{k+1}-\xi= & x_{k}-\xi-\frac{f\left(x_{k}\right)^{2}}{b f\left(x_{k}\right)^{2}+c f\left(y_{k}\right)^{2}} \frac{f\left(x_{k}\right)}{f^{\prime}\left(x_{k}\right)} \\
= & \left(1-\frac{1}{b+(-1+\alpha)^{2} c}\right) e_{k}+\frac{\left(b+\left(1-2 \alpha+3 \alpha^{2}-2 \alpha^{3}\right) c\right) c_{2}}{\left(b+(-1+\alpha)^{2} c\right)^{2}} e_{k}^{2} \\
& +\frac{1}{\left(b+(-1+\alpha)^{2} c\right)^{3}}\left(2(-1+\alpha) \alpha^{2} c\left(b+(-1+\alpha)^{2} c\right) c_{2}^{2}-2\left(b+(-1+\alpha)^{2} c\right)^{2}\left(c_{2}^{2}-c_{3}\right)\right) e_{k}^{3} \\
& +\frac{1}{\left(b+(-1+\alpha)^{2} c\right)^{3}}\left(-\alpha^{2} c\left(\left(-\left(-6+6 \alpha+\alpha^{2}\right) b+3(-1+\alpha)^{2}\left(2-2 \alpha+\alpha^{2}\right) c\right) c_{2}^{2}\right)\right) e_{k}^{3} \\
& +\frac{1}{\left(b+(-1+\alpha)^{2} c\right)^{3}}\left(-2\left(3-4 \alpha+\alpha^{2}\right)\left(b+(-1+\alpha)^{2} c\right) c_{3}\right) e_{k}^{3}+O\left(e_{k}^{4}\right)
\end{aligned}
$$


Now, it is easy to check that the only values of the parameters $b$ and $c$ satisfying that the coefficients of $e_{k}$ and $e_{k}^{2}$ are null correspond to $b=\frac{1-\alpha+2 \alpha^{2}}{2 \alpha^{2}}$ and $c=\frac{1}{2 \alpha^{2}(\alpha-1)}$. Then, the error equation is

$$
e_{k+1}=\frac{\left(-2+2 \alpha+\alpha^{2}\right) c_{2}^{2}+2(-1+\alpha)^{2} c_{3}}{2(-1+\alpha)} e_{k}^{3}+O\left(e_{k}^{4}\right)
$$

and the third-order of convergence of family (2) is proved.

\subsection{The family for nonlinear systems}

The extension to multivariate case of method (2) requires to rewrite the iterative expression in such a way that no functional evaluation of the nonlinear function remain at the denominator, as they will become vectors in the multivariate case. To get this aim, let us consider that, being the first step of the iterative process $y_{k}=x_{k}-\alpha \frac{f\left(x_{k}\right)}{f^{\prime}\left(x_{k}\right)}, f\left(x_{k}\right)$ can be expressed as $f\left(x_{k}\right)=\frac{1}{\alpha}\left(x_{k}-y_{k}\right) f^{\prime}\left(x_{k}\right)$. By using this, the factor of the second step of (2) involving parameters $b$ and $c$ can be rewritten as

$$
\begin{aligned}
\frac{f\left(x_{k}\right)^{2}}{b f\left(x_{k}\right)^{2}+c f\left(y_{k}\right)^{2}} & =\frac{1}{b+c\left(\frac{f\left(y_{k}\right)}{f\left(x_{k}\right)}\right)^{2}} \\
& =\frac{1}{b+c\left(\frac{\alpha f\left(y_{k}\right)}{\left(x_{k}-y_{k}\right) f^{\prime}\left(x_{k}\right)}\right)^{2}} \\
& =\frac{1}{b+c \alpha^{2}\left[\frac{1}{\alpha}-\frac{f\left[y_{k}, x_{k}\right]}{f^{\prime}\left(x_{k}\right)}\right]^{2}} .
\end{aligned}
$$

By using this transformation, method (2) is fully extensible to several variables, being its iterative expression

$$
\begin{aligned}
y^{(k)} & =x^{(k)}-\alpha\left[F^{\prime}\left(x^{(k)}\right)\right]^{-1} F\left(x^{(k)}\right), \quad k=0,1, \ldots \\
x^{(k+1)} & =x^{(k)}-\left[b I+c \alpha^{2}\left(\frac{1}{\alpha} I-\left[F^{\prime}\left(x^{(k)}\right)\right]^{-1}\left[y^{(k)}, x^{(k)} ; F\right]\right)^{2}\right]^{-1}\left[F^{\prime}\left(x^{(k)}\right)\right]^{-1} F\left(x^{(k)}\right),
\end{aligned}
$$

where $\left[y^{(k)}, x^{(k)} ; F\right]$ denotes the divided difference of operator $F$ on $y^{(k)}$ and $x^{(k)}$. Identity matrix is denoted by $I$.

For proving the order of the method in $\mathbb{R}^{n}$, it is necessary to recall the definition given by J.M. Ortega and W.G. Rheinboldt [5] of first divided difference of $F$ on $\mathbb{R}^{n}$ as a mapping $[\cdot, \cdot ; F]: D \times D \subset \mathbb{R}^{n} \times \mathbb{R}^{n} \rightarrow \mathcal{L}\left(\mathbb{R}^{n}\right)$ which satisfies $[x, y ; F](x-y)=F(x)-F(y), \forall x, y \in D$.

From the Genocchi-Hermite formula [6]

$$
[x, x+h ; F]=\int_{0}^{1} F^{\prime}(x+t h) d t,
$$

and by expanding $F^{\prime}(x+t h)$ in Taylor's series around the point $x$, we obtain

$$
\int_{0}^{1} F^{\prime}(x+t h) d t=F^{\prime}(x)+\frac{1}{2} F^{\prime \prime}(x) h+\frac{1}{6} F^{\prime \prime \prime}(x) h^{2}+O\left(h^{3}\right) .
$$

If $e=x-\xi$ and assuming that $F^{\prime}(\xi)$ is nonsingular, we have

$$
\begin{aligned}
F(x) & =F^{\prime}(\xi)\left[e+C_{2} e^{2}+C_{3} e^{3}+C_{4} e^{4}\right]+O\left(e^{5}\right), \\
F^{\prime}(x) & =F^{\prime}(\xi)\left[I+2 C_{2} e+3 C_{3} e^{2}+4 C_{4} e^{3}\right]+O\left(e^{4}\right), \\
F^{\prime \prime}(x) & =F^{\prime}(\xi)\left[2 C_{2}+6 C_{3} e+12 C_{4} e^{2}\right]+O\left(e^{3}\right), \\
F^{\prime \prime \prime}(x) & =F^{\prime}(\xi)\left[6 C_{3}+24 C_{4} e\right]+O\left(e^{2}\right),
\end{aligned}
$$


where $C_{k}=(1 / k !)\left[F^{\prime}(\xi)\right]^{-1} F^{(k)}(\xi), \quad k=2,3, \ldots$

Replacing these expressions in (7) and setting $y=x+h$ and $e_{y}=y-\xi$, we get

$$
[x, y ; F]=F^{\prime}(\xi)\left[I+C_{2}\left(e_{y}+e\right)+C_{3} e^{2}\right]+O\left(e^{3}\right) .
$$

Now, we can establish and prove the following result for nonlinear systems.

Theorem 2. Let $F: D \subseteq \mathbb{R}^{n} \rightarrow \mathbb{R}^{n}$ be a sufficiently differentiable function in an open neighborhood $D$ of $\xi$, that is a solution of the nonlinear system $F(x)=0$. Let us suppose that $F^{\prime}(x)$ is nonsingular in $\xi$ and $x^{(0)}$ is an initial estimation close enough to $\xi$. Then, the sequence $\left\{x^{(k)}\right\}_{k \geq 0}$ obtained by using the iterative expression (6) converges to $\xi$ with order of convergence 3 if $b=\frac{1-\alpha+2 \alpha^{2}}{2 \alpha^{2}}$ and $c=\frac{1}{2 \alpha^{2}(\alpha-1)}$, being $\alpha \notin\{0,1\}$. Then, the error equation of the method is

$$
e^{(k+1)}=\left(\left(\frac{6+6 \alpha-\alpha^{2}}{2(-1+\alpha)}\right) C_{2}^{2}+(\alpha-1)^{2} C_{3}\right) e^{(k)^{3}}+O\left(e^{(k)^{4}}\right)
$$

where $C_{k}=(1 / k !)\left[F^{\prime}(\xi)\right]^{-1} F^{(k)}(\xi), \quad k=2,3, \ldots$, and $e^{(k)}=x^{(k)}-\xi$.

Proof: Taylor expansion of $F\left(x^{(k)}\right)$ and $F^{\prime}\left(x^{(k)}\right)$ around $\xi$ gives us

$$
F\left(x^{(k)}\right)=F^{\prime}(\bar{x})\left[e^{(k)}+C_{2} e^{(k)^{2}}+C_{3} e^{(k)^{3}}\right]+O\left(e^{(k)^{4}}\right),
$$

and

$$
F^{\prime}\left(x^{(k)}\right)=F^{\prime}(\bar{x})\left[I+2 C_{2} e^{(k)}+3 C_{3} e^{(k)^{2}}\right]+O\left(e^{(k)^{3}}\right) .
$$

Moreover, forcing that $\left[F^{\prime}\left(x^{(k)}\right)\right]^{-1} F^{\prime}\left(x^{(k)}\right)=F^{\prime}\left(x^{(k)}\right)\left[F^{\prime}\left(x^{(k)}\right)\right]^{-1}=I$, we obtain

$$
\left[F^{\prime}\left(x^{(k)}\right)\right]^{-1}=\left[I+X_{2} e^{(k)}+X_{3} e^{(k)^{2}}\right]\left[F^{\prime}(\xi)\right]^{-1}+O\left(e^{(k)^{3}}\right),
$$

where

$$
X_{2}=-2 C_{2} \text { and } X_{3}=4 C_{2}^{2}-3 C_{3}
$$

Then,

$$
\left[F^{\prime}\left(x^{(k)}\right)\right]^{-1} F\left(x^{(k)}\right)=e^{(k)}-C_{2} e^{(k)^{2}}+2\left(-C_{3}+C_{2}^{2}\right) e^{(k)^{3}}+O\left(e^{(k)^{4}}\right),
$$

and the error at the first step is

$$
e_{y}^{(k)}=y^{(k)}-\xi=(1-\alpha) e^{(k)}+\alpha C_{2} e^{(k)^{2}}-2 \alpha\left(-C_{3}+C_{2}^{2}\right) e^{(k)^{3}}+O\left(e^{(k)^{4}}\right) .
$$

Now, by using [8],

$$
\left[y^{(k)}, x^{(k)} ; F\right]=F^{\prime}(\xi)\left[I+(2-\alpha) C_{2} e^{(k)}+\alpha C_{2}^{2} e^{(k)^{2}}+\left(3-3 \alpha+\alpha^{2}\right) C_{3} e^{(k)^{2}}\right]+O\left(e^{(k)^{3}}\right)
$$

and then, by applying (11) and (14),

$$
\frac{1}{\alpha} I-\left[F^{\prime}\left(x^{(k)}\right)\right]^{-1}\left[y^{(k)}, x^{(k)} ; F\right]=\left(\frac{1}{\alpha}-1\right) I+\alpha C_{2} e^{(k)}-\left(3 \alpha C_{2}^{2}+\alpha(\alpha-3) C_{3}\right) e^{(k)^{2}}+O\left(e^{(k)^{3}}\right) .
$$

So, the Taylor expansion (around $\xi$ ) of the block to be inverted in the second step of the process is

$$
\begin{aligned}
A= & b I+c \alpha^{2}\left(\frac{1}{\alpha} I-\left[F^{\prime}\left(x^{(k)}\right)\right]^{-1}\left[y^{(k)}, x^{(k)} ; F\right]\right)^{2} \\
= & \left(b+\left(\frac{1}{\alpha}-1\right)^{2} c \alpha^{2}\right) I+2 c \alpha^{3}\left(\frac{1}{\alpha}-1\right) C_{2} e^{(k)} \\
& +\left[c \alpha^{2}\left(\alpha^{2}-6+6 \alpha\right) C_{2}^{2}+\left(2 c \alpha^{3}(\alpha-3)-2 c \alpha^{2}(\alpha-3)\right) C_{3}\right] e^{(k)^{2}}+O\left(e^{(k)^{3}}\right) .
\end{aligned}
$$


Then, we define $A^{-1}=I+Y_{1} e^{(k)}+Y_{2} e^{(k)^{2}}+O\left(e^{(k)^{3}}\right)$ in such a way that $A A^{-1}=A^{-1} A=I$. So, the following expression is forced to become the identity matrix:

$$
\begin{aligned}
A A^{-1}= & \left(b+\left(\frac{1}{\alpha}-1\right)^{2} c \alpha^{2}\right) I+\left[\left(b+\left(\frac{1}{\alpha}-1\right)^{2} c \alpha^{2}\right) Y_{1}+2 c \alpha^{3}\left(\frac{1}{\alpha}-1\right) C_{2}\right] e^{(k)} \\
& +\left[\left(b+\left(\frac{1}{\alpha}-1\right)^{2} c \alpha^{2}\right) Y_{2}+2 c \alpha^{3}\left(\frac{1}{\alpha}-1\right) C_{2} Y_{1}+c \alpha^{2}\left(\alpha^{2}-6+6 \alpha\right) C_{2}^{2}\right. \\
& \left.+\left(2 c \alpha^{3}(\alpha-3)-2 c \alpha^{2}(\alpha-3)\right) C_{3}\right] e^{(k)^{2}}+O\left(e^{(k)^{3}}\right) .
\end{aligned}
$$

In order to get this aim, it is necessary that the following equalities are verified:

$$
\begin{aligned}
b & =1-\left(\frac{1}{\alpha}-1\right)^{2} c \alpha^{2}, \\
Y_{1} & =-2 c \alpha^{3}\left(\frac{1}{\alpha}-1\right) C_{2}, \\
Y_{2} & =-Y_{1}^{2}-c \alpha^{2}\left(\alpha^{2}-6+6 \alpha\right) C_{2}^{2}-\left(2 c \alpha^{3}(\alpha-3)-2 c \alpha^{2}(\alpha-3)\right) C_{3} .
\end{aligned}
$$

Then, the error at the last step is

$$
\begin{aligned}
x^{(k+1)}-\xi= & x^{(k)}-\xi-A^{-1}\left[F^{\prime}\left(x^{(k)}\right)\right]^{-1} F\left(x^{(k)}\right) \\
= & \left(-2 c \alpha^{3}\left(\frac{1}{\alpha}-1\right) C_{2}-C_{2}\right) e^{(k)^{2}} \\
& +\left(-2 C_{3}+2 C_{2}^{2}+2 c \alpha^{3}\left(\frac{1}{\alpha}-1\right) C_{2}^{2}-4 c^{2} \alpha^{6}\left(\frac{1}{\alpha}-1\right)^{2} C_{2}^{2}\right. \\
& \left.-c \alpha^{2}\left(\alpha^{2}-6-6 \alpha\right) C_{2}^{2}-\left(2 c \alpha^{3}(\alpha-3)-2 c \alpha^{2}(\alpha-3)\right) C_{3}\right) e^{(k)^{3}}+O\left(e^{(k)^{4}}\right)
\end{aligned}
$$

and the only way to reach third-order of convergence is to force parameter $c$ to be $c=\frac{1}{2 \alpha^{2}(\alpha-1)}$ and, therefore, $b=\frac{1-\alpha+2 \alpha^{2}}{2 \alpha^{2}}$. Then, the error equation of the method is

$$
e^{(k+1)}=\left(\left(\frac{6+6 \alpha-\alpha^{2}}{2(-1+\alpha)}\right) C_{2}^{2}+(\alpha-1)^{2} C_{3}\right) e^{(k)^{3}}+O\left(e^{(k)^{4}}\right) .
$$

\section{Dynamical behavior}

In the following some dynamical planes corresponding to family (2) and some classical methods on functions

- $f_{1}(x)=\arctan (x), \quad \xi=0$,

- $f_{2}(x)=\arctan (x)-\frac{2 x}{1+x^{2}}, \quad \xi_{1}=0, \xi_{2} \approx-1.3917$ and $\xi_{3} \approx 1.3917$,

- $f_{3}(x)=\frac{x^{2}-1}{x^{2}+1}+1, \quad \xi=0$,

are shown.

In order to study the dynamical behavior of these iterative methods, it is necessary to recall some basic dynamical concepts. For a more extensive review of these concepts, see [7, 8]. 


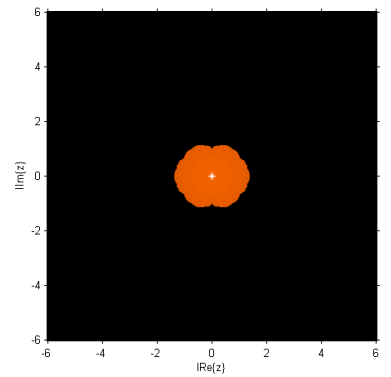

(a) Newton

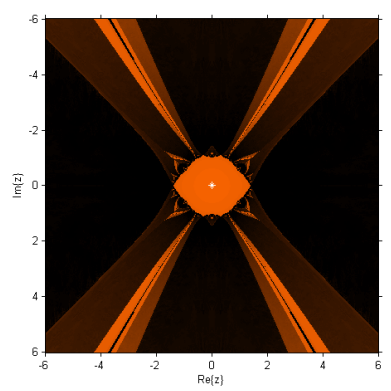

(c) Traub

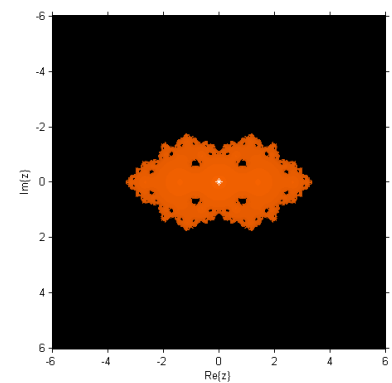

(b) Kalitkin

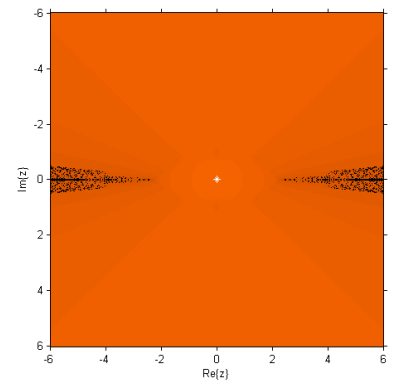

(d) Ostrowski

Figure 1: Dynamical planes of $f_{1}(x)=\arctan (x)$

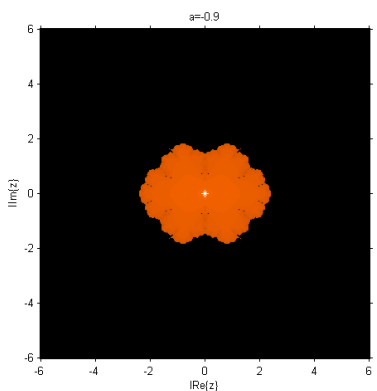

(a) $\alpha=-0.9$

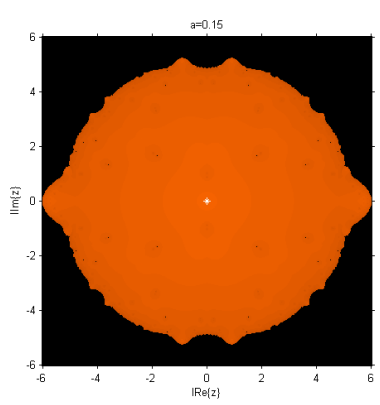

(d) $\alpha=0.15$

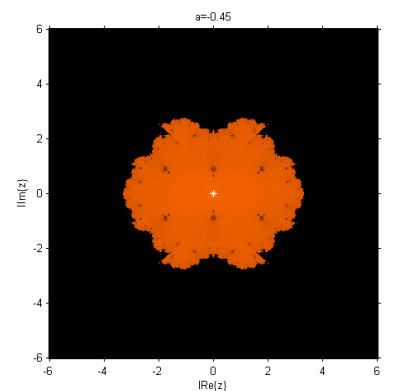

(b) $\alpha=-0.45$

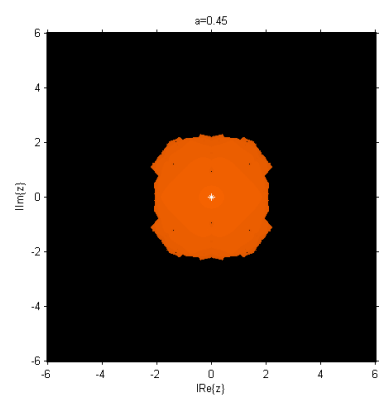

(e) $\alpha=0.45$

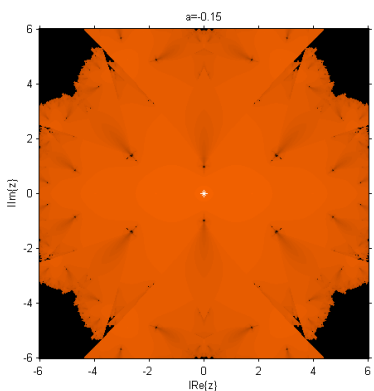

(c) $\alpha=-0.15$

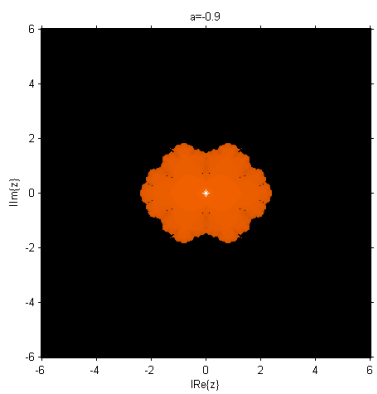

(f) $\alpha=0.9$

Figure 2: Dynamical planes of the proposed methods on $f_{1}(x)=\arctan (x)$ 
Let $R: \hat{\mathbb{C}} \rightarrow \hat{\mathbb{C}}$ be an operator that results from applying an iterative method on a particular function, where $\hat{\mathbb{C}}$ is the Riemann sphere. The orbit of a point $z_{0} \in \hat{\mathbb{C}}$ is defined as the set of successive images of $z_{0}$ by the operator, $\left\{z_{0}, R\left(z_{0}\right), \ldots, R^{n}\left(z_{0}\right), \ldots\right\}$.

The dynamical behavior of the orbit of a point on the complex plane can be classified depending on its asymptotic behavior. In this way, a point $z_{0} \in \mathbb{C}$ is a fixed point of $R$ if $R\left(z_{0}\right)=z_{0}$. A fixed point is attracting, repelling or neutral if $\left|R^{\prime}\left(z_{0}\right)\right|$ is lower than, greater than or equal to 1, respectively. Moreover, if $\left|R^{\prime}\left(z_{0}\right)\right|=0$, the fixed point is superattracting.

If $z_{f}^{*}$ is an attracting fixed point of the operator $R$, its basin of attraction $\mathcal{A}\left(z_{f}^{*}\right)$ is defined as the set of pre-images of any order such that

$$
\mathcal{A}\left(z_{f}^{*}\right)=\left\{z_{0} \in \hat{\mathbb{C}}: R^{n}\left(z_{0}\right) \rightarrow z_{f}^{*}, n \rightarrow \infty\right\} .
$$

The set of points whose orbits tends to an attracting fixed point $z_{f}^{*}$ is defined as the Fatou set, $\mathcal{F}(R)$. The complementary set, the Julia set $\mathcal{J}(R)$, is the closure of the set of pre-images of its repelling fixed points, and establishes the boundaries between the basins of attraction.

For the representation of the convergence basins of our procedures and classical methods, we have used the software described in [9]. We draw a mesh with four hundred points per axis; each point of the mesh is a different initial estimation which we introduce in each procedure. In case of $f_{1}(x)$ and $f_{3}(x)$, which have only one root, when the method reaches the solution in less than eighty iterations, this point is drawn in orange (in case of $f_{2}(x)$, that has three different solutions, the second and third basins are painted in green and blue). The color will be more intense when the number of iterations is lower. Otherwise, if the method arrives at the maximum of iterations, the point will be drawn in black.

Under these conditions, some elements of the family of proposed methods are analyzed, and compared with Newton', Kalitkin', Traub' and Ostrowski's schemes. In Figure 1, the basins of attraction of these methods on $f_{1}(x)$ are observed, and it can be noticed that the widest basin of attraction correspond to the last one, in the complex plane. If we pay attention to reals, the interval of convergence of Kalitkin's algorithm is $[-3.5,3.5]$, approximately, meanwhile the ones of Newton' and Traub's methods are both $[-1.8,1.8]$, approximately and for Ostrowski's scheme it would be $[-2.5,2.5]$.

We can see at Figure 2 that, by using PM method, the widest basins of attraction correspond to values of the parameter $\alpha$ close to zero (Figures $2 \mathrm{c}$ and $2 \mathrm{~d}$ ) with real regions of convergence bigger than $[-6,6]$. Moreover, in terms of real initial estimations with convergence to the roots, the intervals of convergence are bigger than the one of Newton's in all cases.

In case of $f_{2}(x)$, let us notice that, when method (2) is used with different values of $\alpha$ (see Figure 4), the basins of attraction of the three solutions of the equation appear in blue, orange and green. In case of Newton's scheme (see Figure 3a), it can be observed that these areas of convergence are bounded in the plotted region, $[-6,6] \times[-6,6]$. Nevertheless, the small black regions do not correspond to strange fixed points, but to pre-images of the infinity. When Traub's method is used (see Figure 3c), the black basin (convergence to the infinity) is "crossed" by orange regions of convergence to zero. Moreover, the pre-images of the infinity inside the bounded region of convergence are bigger than in case of Newton's. In Kalitkin's case, the real initial estimations with convergence to the roots can be very far from them, but its dynamics is very complicated. However, the dynamical plane associated to Ostrowski's procedure is clean and the basins are wider than in previous cases. Indeed, there are no convergence to the infinity.

When the dynamical behavior of the elements of the new family is observed on $f_{2}(x)$, again the stability of the methods seems to be directly related with the absolute value of the parameter. In fact, the best results are obtained for $\alpha=-0.15$ (Figure $4 \mathrm{c}$ ) and $\alpha=0.15$ (Figure 4d), being the first of these cases much more stable than classical methods as Newton, Traub or Ostrowski.

Finally, let us consider the function $f_{3}(x)=\frac{x^{2}-1}{x^{2}+1}+1$. In Figures 5 and 6 we can see the basins of attraction of the unique root $\xi=0$, for different classical methods (Figure 5) and for the proposed schemes, and different values of the parameter $\alpha$ (Figure 6). We can observe that, again in this case, widest basins are found for 


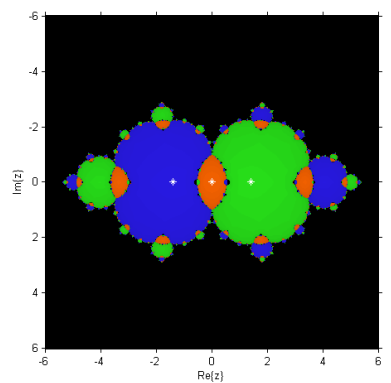

(a) Newton

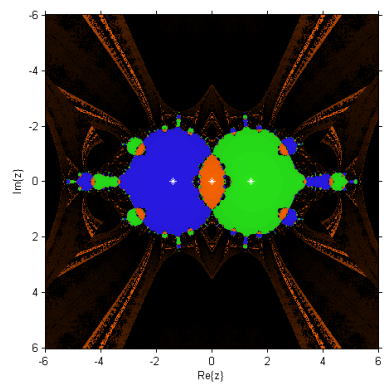

(c) Traub

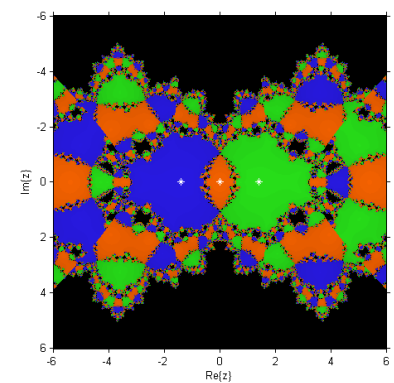

(b) Kalitkin

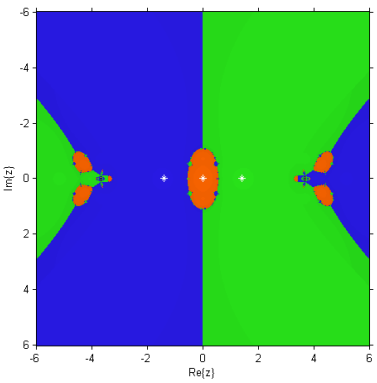

(d) Ostrowski

Figure 3: Dynamical planes of $f_{2}(x)=\arctan (x)-\frac{2 x}{1+x^{2}}$

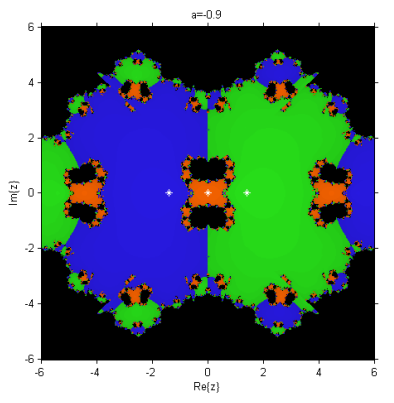

(a) $\alpha=-0.9$

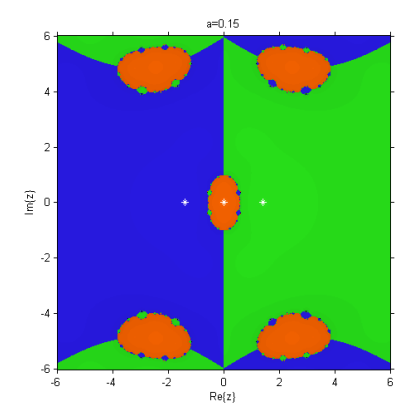

(d) $\alpha=0.15$

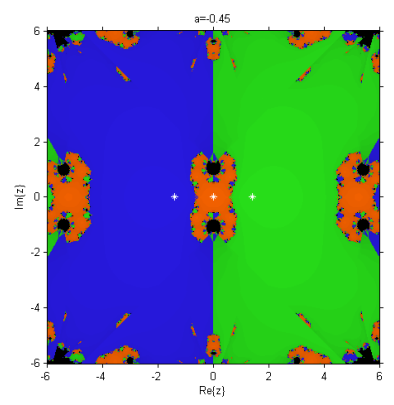

(b) $\alpha=-0.45$

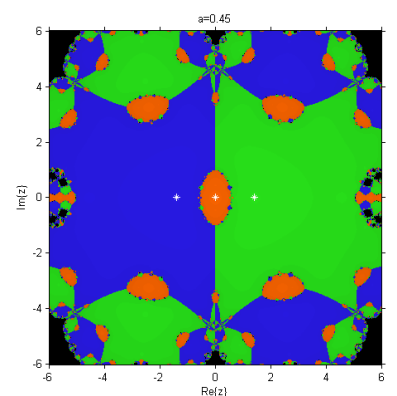

(e) $\alpha=0.45$

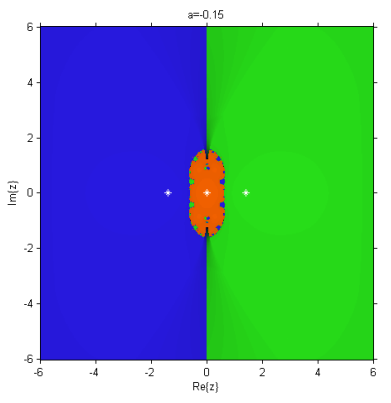

(c) $\alpha=-0.15$

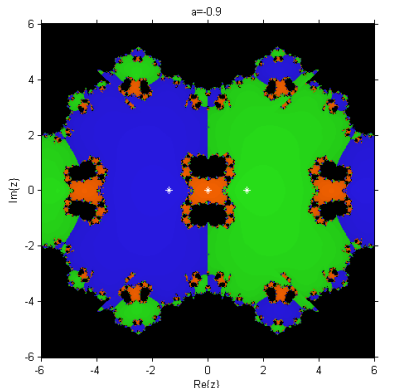

(f) $\alpha=0.9$

Figure 4: Dynamical planes of the proposed methods on $f_{2}(x)=\arctan (x)-\frac{2 x}{1+x^{2}}$ 


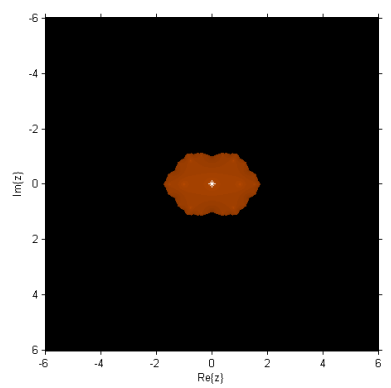

(a) Newton

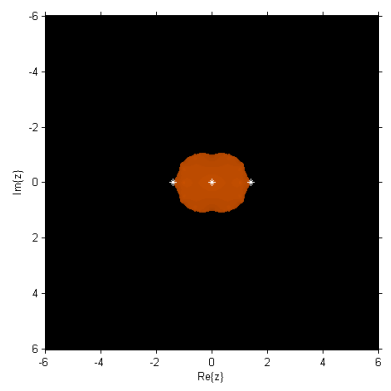

(c) Traub

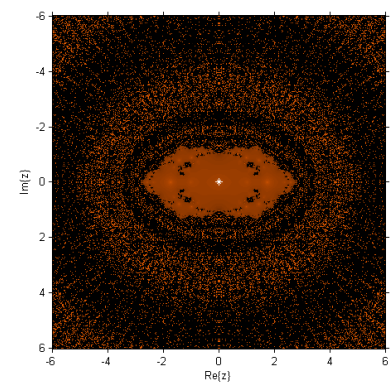

(b) Kalitkin

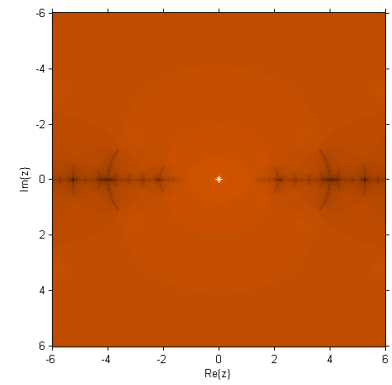

(d) Ostrowski

Figure 5: Dynamical planes of $f_{3}(x)=\frac{x^{2}-1}{x^{2}+1}+1$

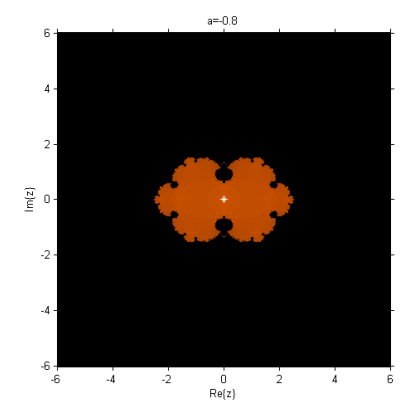

(a) $\alpha=-0.8$

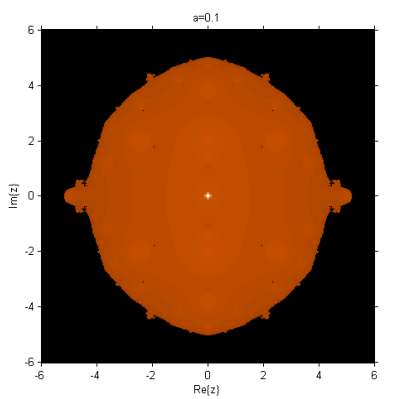

(d) $\alpha=0.1$

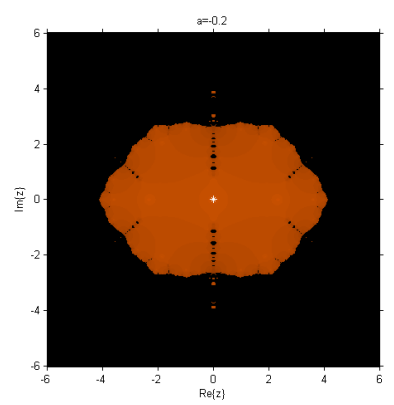

(b) $\alpha=-0.2$

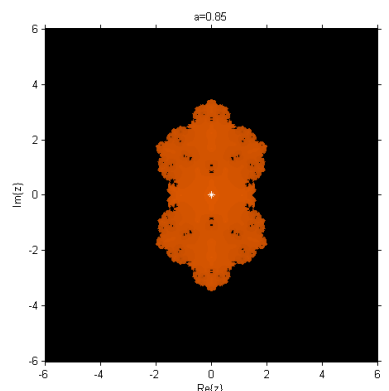

(e) $\alpha=0.85$

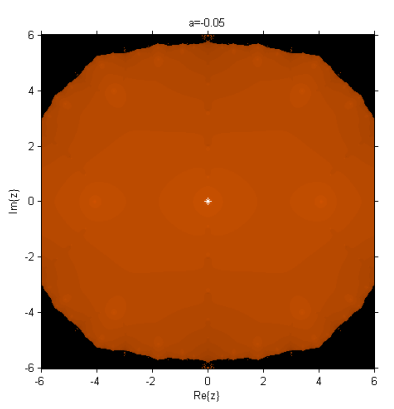

(c) $\alpha=-0.05$

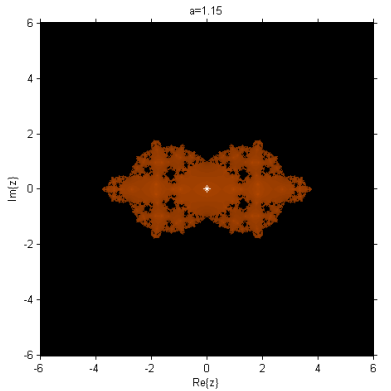

(f) $\alpha=1.15$

Figure 6: Dynamical planes of the proposed methods on $f_{3}(x)=\frac{x^{2}-1}{x^{2}+1}+1$ 
small values of the parameter (in absolute value). In this case, Figures 6c and 6d correspond to the more stable methods. Nevertheless, it can be observed in Figure 6f that bigger values of $\alpha$ can have also a big interval of convergence (in real cases), as it happens for $\alpha=1.15$, for example.

\section{Numerical results}

Numerical computations have been carried out by using variable precision arithmetic in Mathematica 9 with 10.000 significant digits. The tolerance is set up to $10^{-2000}$ in all numerical tests.

Below we provide the results of the numerical experiment that we carried out in order to compare Newton', Traub', Kalitkin', Ostrowski's and proposed method (PM) 22. We use the same functions $f_{1}(x), f_{2}(x), f_{3}(x)$ as in Section 3. We present the obtained results in Table 1, where one can see the relations between the zero estimate and the number of iterations for different equations.

In Table 1 one can see some data that help to compare the methods, as the number of iterations iter and the Approximated Computational Order of Convergence ACOC given by (see [10]):

$$
p \approx A C O C=\frac{\ln \left(\left\|x_{k+1}-x_{k}\right\| /\left\|x_{k}-x_{k-1}\right\|\right)}{\ln \left(\left\|x_{k}-x_{k-1}\right\| /\left\|x_{k-1}-x_{k-2}\right\|\right)} .
$$

In all examples one can observe wider convergence domain of PM method (2), while other iterative schemes appear to be inefficient. Values of parameter $\alpha$ are chosen $0.15,-0.15,-0.05$ for equations $f_{1}(x), f_{2}(x), f_{3}(x)$ correspondingly, as they show better dynamical behavior (see Section 3 ).

In order to read into the ACOC results in Table 1, let us remark that the error equation of Newton', Kalitkin', Traub' and Ostrowski's methods depend only on $c_{2}=\frac{1}{2} \frac{f^{\prime \prime}(\xi)}{f^{\prime}(\xi)}$. So, the increased ACOC that appears in case of function $f_{1}(x)$ is due to the fact that the second derivative of this function is null at the solution. When function $f_{2}(x)$ is considered, the numerical results for ACOC correspond to the theoretical order of convergence, if they converge. Finally, for function $f_{3}(x)$ the solution is a multiple root and all the methods have linear convergence, when they converge.

On the other hand, we can observe that PM scheme needs lower number of iterations than Traub's method, and has wider convergence domain than all the classical methods used in this work.

Now we present the results of numerical tests for the system of equations, known as Brown quasi-linear system [2], described by

$$
\begin{gathered}
f_{i}(x)=x_{i}+\sum_{j=1}^{N} x_{j}-N-1, \quad 1 \leq i \leq N-1, \\
f_{N}(x)=\prod_{j=1}^{N} x_{j}-1 .
\end{gathered}
$$

This system has different solutions including a trivial one $(1,1 \ldots 1)$, but we use only zero estimates that reduce all methods to the same solution, in order to carry out "fair" comparison.

We excluded Ostrowski's method from the comparison because it has nontrivial generalization to systems of equations. The dimension of system (16) is set up to 4 and 6. Corresponding numerical results for certain zero estimates are shown in Table 2 . Note that Newton's and Kalitkin's methods have quadratic convergence rate while PM and Traub's methods have cubic convergence.

\section{Conclusions}

In this paper we have introduced a new parametric family of third-order iterative methods both for equations and systems of equations. The main aim was to extend the convergence domain. We have carried out dynamical study and numerical tests in order to compare the proposed method with methods of Newton, Kalitkin, Traub and Ostrowski. Theoretical results coincide with the results that were obtained during numerical experiment and show advantages of proposed class of methods as compared with well-known iterative schemes. 


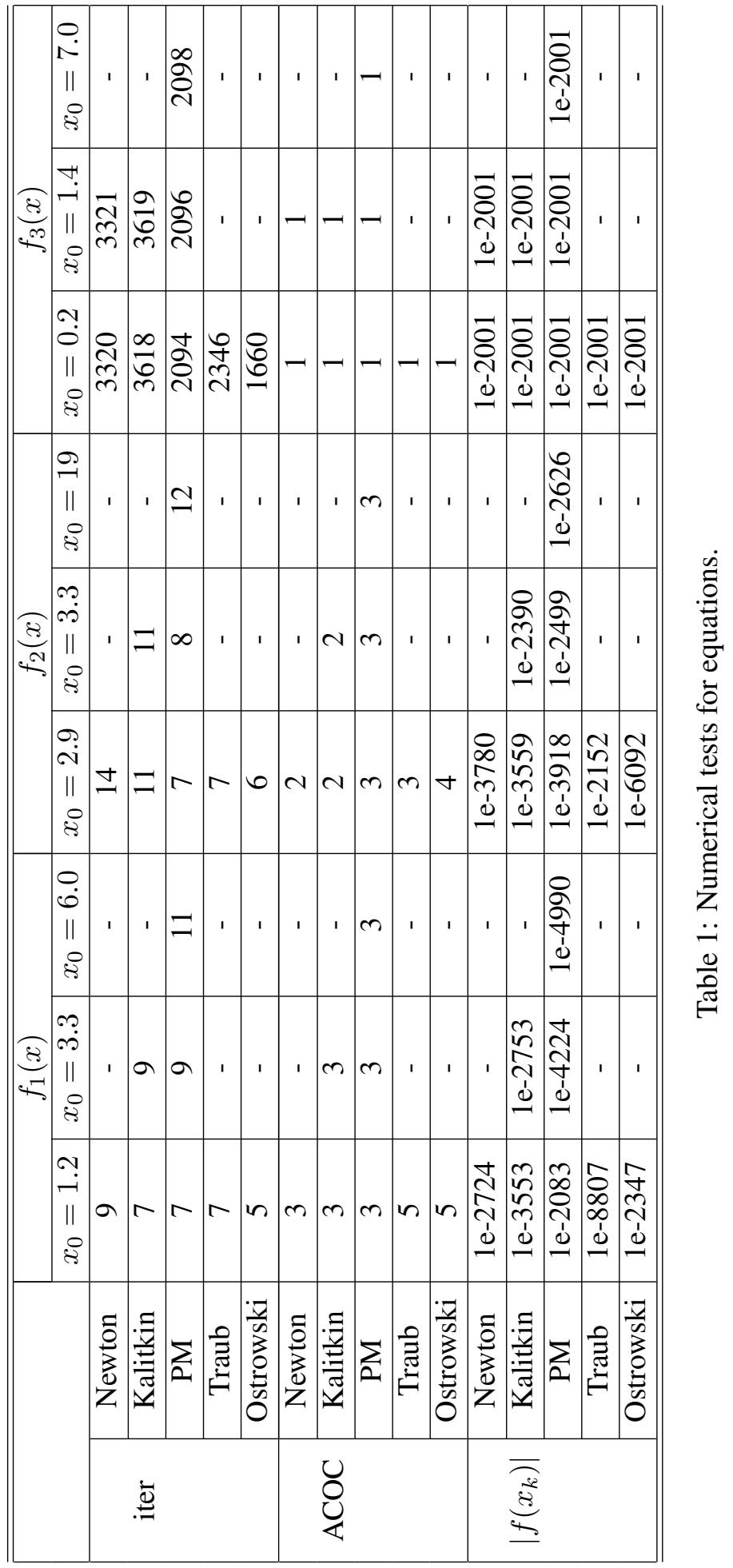




\begin{tabular}{|c|c|c|c|c|c|}
\hline & \multicolumn{4}{|c|}{ Number of iterations } \\
\hline & & Newton & Kalitkin & PM & Traub \\
\hline \multirow{3}{*}{$N=4$} & $x^{(0)}=(-44,91,69,9)^{T}$ & 27 & - & 9 & - \\
\hline & $x^{(0)}=(-99.8,13.3,-85.2,-52.6)^{T}$ & 26 & 28 & 10 & 19 \\
\hline & $x^{(0)}=(-58.8,-31.3,60.9,-52.9)^{T}$ & 30 & 32 & 9 & 44 \\
\hline \multirow{3}{*}{$N=6$} & $x^{(0)}=(9.9,7.3,-5.2,-8.8,6.5,-0.2)^{T}$ & 17 & 18 & 9 & 12 \\
\hline & $x^{(0)}=(-3.96,-6,0.88,3.46,-7,-4.13)^{T}$ & 23 & 24 & 10 & 17 \\
\hline & $x^{(0)}=(3.45,5.34,-6.67,4.39,-7,1.3)^{T}$ & 52 & - & 10 & - \\
\hline
\end{tabular}

Table 2: Numerical tests for system $(16)$.

\section{References}

[1] M. Petković, B. Neta, L. Petković and J. Džunić, Multipoint methods for solving nonlinear equations, Academic Press, 2012.

[2] V.V. Ermakov, N. N. Kalitkin, The optimal step and regularization for Newton's method, USSR Computational Mathematics and Mathematical Physics, 21(2) (1981) 235-242.

[3] J. F. Traub, Iterative methods for the solution of equations, Prentice-Hall, Englewood Cliffs, 1964.

[4] A.M. Ostrowski, Solutions of equations and systems of equations, Academic Press, New York-London, 1966.

[5] Ortega J.M., Rheinboldt W.G., Iterative solutions of nonlinear equations in several variables, Academic Press, New York, 1970.

[6] Hermite, C., Sur la formule d'interpolation de Lagrange, J. Reine Angew. Math., 84 (1878) 70-79.

[7] P. Blanchard, The dynamics of Newton's method, Proc. of Symposia in Applied Math., 49 (1994) 139154.

[8] R.L. Devaney, The Mandelbrot Set, the Farey Tree and the Fibonacci sequence, Am. Math. Monthly, 106(4) (1999) 289-302.

[9] F.I. Chicharro, A. Cordero, Juan R. Torregrosa, Drawing Dynamical and Parameters Planes of Iterative Families and Methods, The Scientific World Journal 2013, Article ID 780153, 11 pages.

[10] A. Cordero, J.R. Torregrosa. Variants of Newton's Method using fifth-order quadrature formulas. Applied Mathematics and Computation, 190 (2007) 686-698. 